\title{
Vegetationsdynamik auf Moränendauerflächen des Grossen Aletschgletschers im Wallis

\author{
Erste Ergebnisse aus den Neuaufnahmen der 54-jährigen Flächen von LÜDI
}

\section{Silvan Rehberger, Freiburg im Breisgau}

\section{Sukzession auf Moränenflächen}

Seit dem Ende der neuzeitlichen Gletscherhochstandsphase im 19. Jahrhundert schmelzen die Zungenregionen der meisten alpinen Gletscher zurück. Die auf den freigegebenen Moränenflächen stattfindende pflanzliche Primärsukzession ist bereits seit langem Gegenstand botanischer Untersuchungen. Erste Schilderungen stammen unter anderem von COAZ (1887) für die Moränen des Rhonegletschers.

Die ältesten Dauerflächen auf neuzeitlichen Moränenflächen wurden bereits 1916 von COOPER (1923) in der Glacier Bay in Alaska angelegt. Seither sind weltweit zahlreiche Publikationen zur pflanzlichen Primärsukzession auf Moränen erschienen. Einen guten Überblick hierüber gibt MarTHEws (1992).

Methodisch sind zwei weit verbreitete Ansätze in der Erforschung der Primärsukzession zu unterscheiden: die zu den direkten Methoden zählende Arbeit mit Dauerbeobachtungsflächen und die zu den indirekten Methoden zählende Arbeit mit der Chronosequenz im Sinne einer «space-for-time substitution» (PICKETT 1989).

Für die Alpen liegen eine ganze Reihe aktueller Untersuchungen vor, so zum Beispiel von BöHMER (1999), Burga (1999), Egger (1997), Erschbamer et al. (1997), Erschbamer et al. (1999), Krause \& Frenzel (1997), SChubiger-Bossard (1988) und anderen. Theurillat (1992) diskutiert einen symphytocoenologischen Ansatz, Richter (1994) geht auf Störungen innerhalb von Sukzessionsprozessen ein, STÖcKLIN \& BäUmler (1996) legen einen Schwerpunkt auf Diasporeneintrag und Etablierungsstrategien und Erschbamer (1997) betrachtet Aspekte des «global change» im Zusammenhang mit Gletschervorfeldern.

Der vorliegende Beitrag stellt Ergebnisse der 1998 erfolgten pflanzensoziologischen Neufnahme auf den 1944 von LüDI (1945) angelegten Dauerflächen auf Moränen des Grossen Aletschgletschers dar. Da es sich hierbei um ein noch nicht abgeschlossenes Projekt handelt, fällt die Diskussion der Ergebnisse eher kurz aus.

\section{Das Untersuchungsgebiet}

Das Untersuchungsgebiet ist Bestandteil des 1933 unter Schutz gestellten Naturreservates «Aletschwald», gelegen an der nordwestlich exponierten Hangseite am Zungenende des Grossen Aletschgletschers (Kanton Wallis) in der Schweiz (Karte 1). Über die Vegetation des Reservates liegt eine Karte mit ausführlichen Erläuterungen von Richard (1968) vor. Die Moosflora und Moosvegetation beschreibt BERTRAM (2000).

Etwa 180 Hektar sind dem Larici-Pinetum cembrae (Lärchen-Arvenwald) zuzurechnen, 25 Hektar dem Piceetum subalpinum (subalpiner Fichtenwald) und 40 Hektar einer offenen Mosaikvegetation aus EmpetroVaccinietum (Rauschbeer-Krähenbeerenheide), Salicetum herbaceae (Krautweiden-Schneetälchen) und einem sehr lückigem Larici-Pinetum cembrae. Ein rund 175 Hektar grosser Teil des Reservates besteht aus jungen Moränenflächen im Höhenbereich zwischen etwa 1700 und $1950 \mathrm{~m} \mathrm{NN}$.

Ihre Entstehung datiert auf den Rückzug des Grossen Aletschgletschers seit dem Ende seines letzten Höchststandes zwischen 1856 und 1860 (vgl. dazu Holzhauser 1984 und 1995). Das stetige Abschmelzen der Zungenregion des Gletschers führt zu einer Vergrösserung des Schutzgebietes um einen mehrere Meter breiten Streifen pro Jahr. Das die Moränen bildende Gesteinsmaterial ist vorwiegend silikatischen Ursprungs und lässt sich unter anderem den für das Gebiet typischen Biotitgneisen zuordnen. Daneben sind dunkelgraue geschieferte Kalke aus der MönchJungfrau-Region unregelmässig eingestreut. Abhängig von Gletschertätigkeit und Relief des Ausgangsgesteines sind die Moränen in kleine Wälle, Verflachungen, Mulden oder Rinnen sowie Hänge bis $30^{\circ}$ Neigung gegliedert.

\section{Die Dauerflächen}

Im Jahr 1944 legte WERNER LÜDI im Lärchen-Arvenwald und auf den jungen Moränen des Reservates Aletschwald eine Reihe von Dauerbeobachtungsflächen an. Sie gehören zu den ältesten alpinen Dauerflächen auf Moränen. Insgesamt wies er sechs Kleinflächen bis 30 qm Grösse und sechs Grossflächen bis 800 qm Grösse aus. Die Kleinflächen liegen auf dem Kamm der Jungmoräne aus dem Jahr 1859/1860, die grossen 


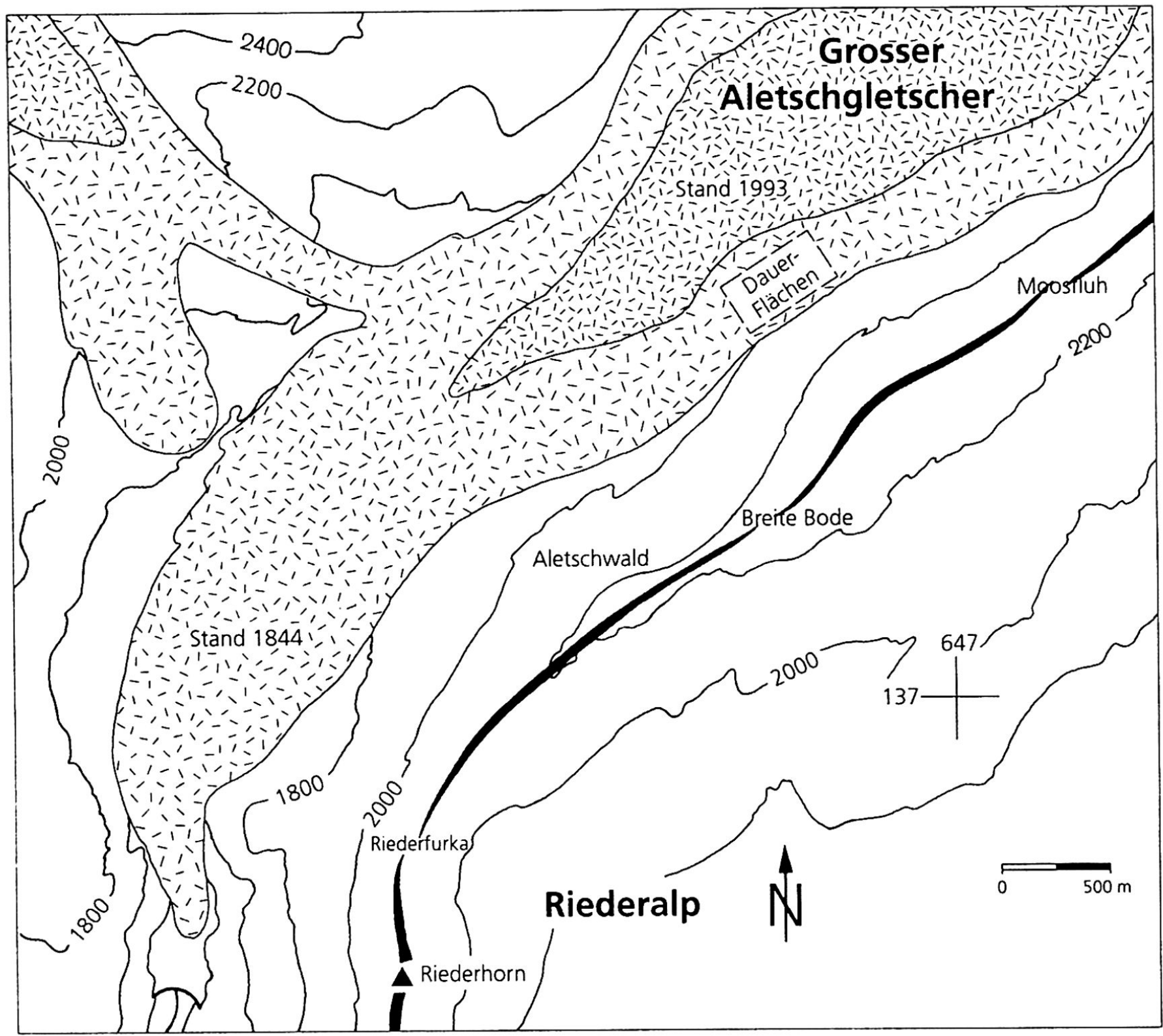

Karte 1: Lage des Untersuchungsgebietes am Rande des Grossen Aletschgletschers (Wallis, Schweiz). Die Gletscherausdehnung ist für die Stadien von 1844 und 1993 eingetragen.

Map of the study area at the Great Aletsch Glacier (Valais, Switzerland). Glacier boundaries are drawn for 1844 and 1993.

Carte du périmètre étudié au "Grosser Aletschgletscher» (Valais, Suisse). L'extension du glacier est mentionnée pour les années 1844 et 1993.

Quelle: Karte verändert und aktualisiert nach Holzhauser (1988); Kartographie: L. BaumanN

Flächen auf durch das anstehende Gestein gebildeten Terrassen, auf denen das Moränenmaterial abgelagert ist.

Neben pflanzensoziologischen Aufnahmen führte LüDI (1945, 1950) auch detaillierte, nach Höhenklassen getrennte Gehölzzählungen aller vertretenen Baumarten durch.
JeAn-Louis Richard (1973) griff diese Untersuchungen auf. Unter seiner Betreuung wurden die pflanzensoziologischen Aufnahmen in den Jahren 1971 (Kretz 1972) und 1981 (Morier-Genoud 1982) wiederholt. Ausserdem richtete er weitere Dauerflächen auf neu freigegebenem Moränensubstrat ein, für die er die Sukzession über 15 Jahre hinweg in Richard (1987) beschrieb. 


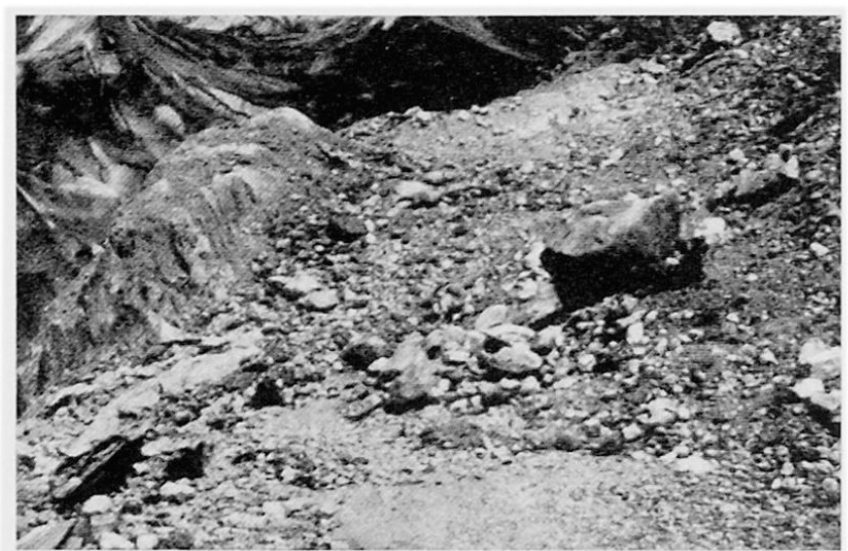

Abb. 1: Blick auf einen Teil der Dauerfläche MD 1 im Jahr 1944. Im Hintergrund ist der Eiskörper des Gletschers zu sehen.

Part of the permanent plot MD 1 in 1944. In the background the ice-masses of the Great Aletsch Glacier may bee seen.

Aperçu d'une partie de la surface permanente MD I en 1944. A l'arrière-plan la masse du glacier est visible.

Quelle: LÜDI (1945); Abdruck mit freundlicher Genehmigung des Geobotanischen Instituts der ETH Zürich

Während die Dauerflächen im Bereich des Aletschwaldes 1992 und 1993 neu bearbeitet wurden (EGGENBERG 1996), fanden seit 1981 keine pflanzensoziologischen Aufnahmen und seit 1971 keine Gehölzzählungen in den Dauerflächen von LüDı mehr statt. Dies war Anlass für die Wiederaufnahme und die Erweiterung der Untersuchungen im Jahr 1998, deren Umfang und erste Ergebnisse im Folgenden dargestellt werden. Abb. 1 und Abb. 2 geben einen exemplarischen Eindruck von den Veränderungen auf den Dauerflächen seit 1944.

\section{Umfang der Untersuchungen}

Das Wiederauffinden der Flächen von LüDı war dank seiner genauen Beschreibungen und den zumeist auch nach 50 Jahren gut sichtbaren Markierungen mit Lärchenkanthölzern in weiten Teilen unproblematisch. Von den Kleinflächen ging eine dauerhaft verloren, zwei weitere Kleinflächen konnten rekonstruiert werden. Die sechs grossen Flächen überstanden die Zeit unbeschadet. Die von Richard in einem Hang angelegte Fläche $M$ verlor zwei der vier Begrenzungspfosten, konnte aber nach Rücksprache rekonstruiert werden.

Über einen Zeitraum von zweieinhalb Monaten wurden alle Flächen im Sommer 1998 pflanzensoziologisch neu aufgenommen. In den seit 1981 vom

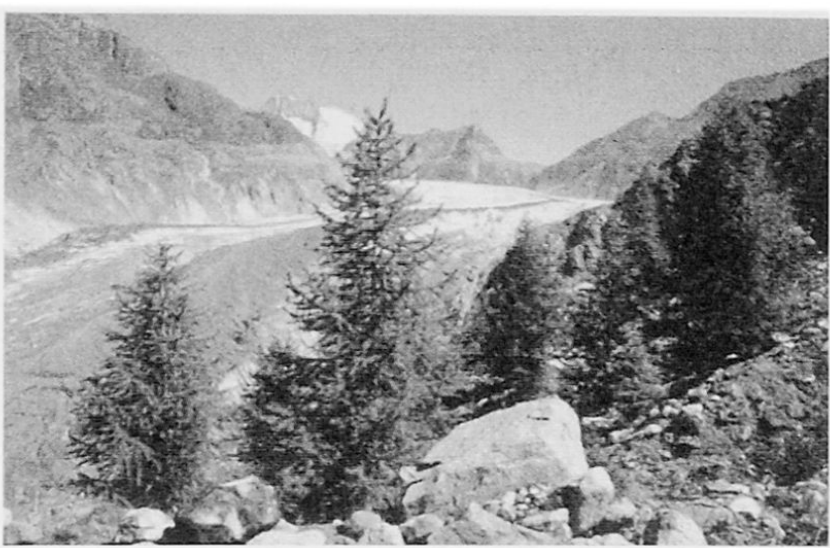

Abb. 2: Blick auf einen Teil der Dauerfläche MD 1 im Jahr 1998. Im Bereich hinter der Moränenfläche ist das Substrat abgerutscht und umgelagert. Bis zu $6 \mathrm{~m}$ hohe Lärchen prägen heute das Bild der Fläche.

Part of the permanent plot MD 1 in 1998. The moraines were partly shifted by the melting ice. "Larix decidua" of heights up to 6 metres dominate the scene today. Aperçu d'une partie de la surface permanente MD 1 en 1998. Dans le domaine situé à l'arrière de la surface morainique le substrat a glissé vers le bas et s'est relocalisé. Des "Larix decidua" atteignant jusqu'à $6 \mathrm{~m}$ de hauteur caractérisent actuellement l'image de cette surface. Foto: S. REHBERGER

Gletscher freigegebenen Moränenbereichen wurden neue Dauerflächen eingerichtet, um die Kontinuität der Untersuchungen sicherzustellen.

Abb. 3 zeigt eine fotografische Übersicht aller in dieser Arbeit ausgewerteten Dauerflächen auf Moränen und ihre ungefähre Lage innerhalb des Untersuchungsgebietes. In Tab. 1 sind für die in Abb. 3 eingetragenen Flächen, die Meereshöhe und das Jahr der Eisfreigabe angegeben sowie die Jahre, aus denen floristische Daten vorliegen. Parallel zu den pflanzensoziologischen Aufnahmen wurden die Gehölzzählungen in allen vorhandenen Flächen wiederholt, welche allerdings noch nicht ausgewertet sind.

Dieser Artikel beschränkt sich daher auf die Darstellung des floristischen Datensatzes der Dauerflächenbeobachtung aus den Jahren 1944, 1948, 1971, 1981 und 1998.

\section{Auswertung}

Der floristische Datensatz aller Aufnahmen wurde von Hand reduziert: Nur in einer oder zwei Flächen auftretende Arten blieben ebenso unberücksichtigt wie zwei in Tab. 1 nicht aufgeführte Kleinflächen, da es sich bei ihnen um Rasenflächen auf der 140 Jahre alten Moräne handelt, die keine grössere Veränderungstendenz zeig- 


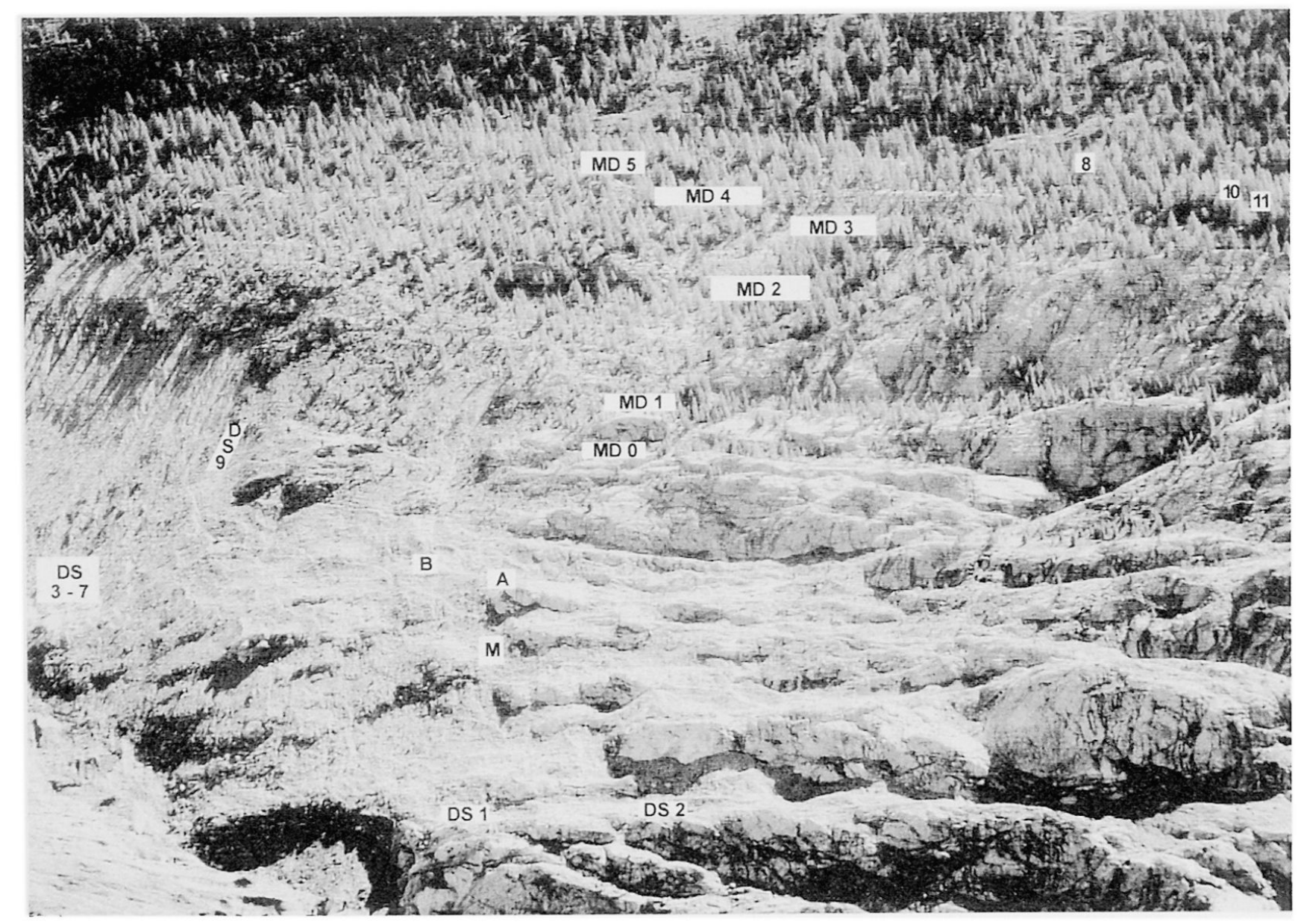

Abb. 3: Übersichtsfoto des Untersuchungsgebietes und ausgewerteter Dauerflächen. Die Bezeichnungen entsprechen Tabelle 1.

Overview of the research area and the permanent plots investigated. The labels are described in Table 1.

La photographie donne un aperçu du périmètre de recherche et surfaces permanentes évaluées. Les dénominations correspondent au tableau 1.

Foto: S. REHBERGER

ten. In die weitere Auswertung gelangte ein Datensatz von 51 Aufnahmen mit insgesamt 170 Arten.

LüDı verwendete für seine Arbeit eine eigene Artmächtigkeits-Skala, die sich von der Skala nach BraunBLANQUET (1928) durch eine zusätzliche Auftrennung der Stufe $2(>5$ bis $=25 \%)$ bei $12.5 \%$ unterschied, wie sie auch BARKMAN et al. (1964) später vorgeschlagen haben. Diese Skala wurde auch 1998 benutzt. Da jedoch 1971 und 1981 keine Trennung der Stufe 2 in 2a und $2 \mathrm{~b}$ erfolgte, wurden in dem gemeinsamen Datensatz die Stufen $2 a$ und $2 b$ wieder zusammengelegt.

Mit Hilfe des Programmpaketes ADE-4 (Thioulouse et al. 1997, 1998), das Module für die multivariate Analyse ökologischer Daten enthält, wurde eine Standard-Korrespondenzanalyse (auch Reciprocal Averaging genannt) ohne Transformation und ohne Entzerrung durchgeführt.
Die Methode der Korrespondenzanalyse ist ein gebräuchliches Verfahren zur Ordination von ökologischen Daten, die einen dominierenden, sehr langen Gradienten enthalten, da ihr ein unimodales Antwortmodell zugrunde liegt. Für Daten, die einen zweiten sehr starken Gradienten enthalten, ist die Methode nicht geeignet.

Die Korrespondenzanalyse liefert für den multidimensionalen Datensatz aus pflanzensoziologischen Aufnahmen eine Projektion auf wenige Achsen, die zu einer maximalen Dispersion der in ihnen auftretenden Pflanzenarten führen, wobei die Artenwerte gewichtete Mittel der Aufnahmewerte sind und umgekehrt. Durch die gleichzeitige Ordination von Arten und Aufnahmen erhält man ein Diagramm gleicher Skalierung der Koordinaten von Arten und Aufnahmen. Dabei liegen die Arten im Schwerpunkt der Aufnahmen, in denen sie auftreten, und zugleich liegen die 


\begin{tabular}{|l|l|l|l|}
\hline Fläche & Höhe (NN) & eisfrei seit & Daten für \\
\hline MD 11 & $1940 \mathrm{~m}$ & 1860 & 1944,1998 \\
MD 10 & $1940 \mathrm{~m}$ & 1860 & 1944,1998 \\
MD 8 & $1965 \mathrm{~m}$ & 1860 & $1944,1971,1998$ \\
MD 5 & $1970 \mathrm{~m}$ & 1860 & $1944,1971,1981,1998$ \\
MD 4 & $1950 \mathrm{~m}$ & 1875 & $1944,1971,1981,1998$ \\
MD 3 & $1930 \mathrm{~m}$ & 1900 & $1944,1971,1981,1998$ \\
MD 2 & $1900 \mathrm{~m}$ & 1920 & $1944,1971,1981,1998$ \\
MD 1 & $1860 \mathrm{~m}$ & 1939 & $1944,1948,1971,1981,1998$ \\
MD 0 & $1850 \mathrm{~m}$ & 1945 & $1948,1971,1981,1998$ \\
MD A & $1805 \mathrm{~m}$ & 1967 & $1971,1981,1998$ \\
MD B & $1800 \mathrm{~m}$ & 1967 & $1971,1981,1998$ \\
MD M & $1780 \mathrm{~m}$ & 1973 & $1981,1986,1998$ \\
DS 1 & $1740 \mathrm{~m}$ & 1985 & 1998 \\
DS 2 & $1750 \mathrm{~m}$ & 1985 & 1998 \\
DS 3 & $1770 \mathrm{~m}$ & 1990 & 1998 \\
DS 4 & $1770 \mathrm{~m}$ & 1990 & 1998 \\
DS 5 & $1770 \mathrm{~m}$ & 1990 & 1998 \\
DS 6 & $1770 \mathrm{~m}$ & 1990 & 1998 \\
DS 7 & $1770 \mathrm{~m}$ & 1990 & 1998 \\
DS 8 & $1780 \mathrm{~m}$ & 1973 & 1998 \\
DS 9 & $1855 \mathrm{~m}$ & 1940 & 1998 \\
\hline
\end{tabular}

Tab.1: Bezeichnungen, Meereshöhe und Jahr der Eisfreigabe der in dieser Arbeit ausgewerteten Moränenflächen sowie Jahre, für welche pflanzensoziologische Aufnahmen vorliegen.

Plot name and corresponding altitudes; years in which the plots became ice-free and the years for which phytosociological data exist.

Dénominations, altitude et année de disparition de la glace des surfaces morainiques étudiées dans le présent article, ainsi que l'indication des années pour lesquelles nous disposons de données phytosociologiques.

Aufnahmen an den Zentroiden der Arten, die in ihnen vorkommen. Der Vorteil der Korrespondenzanalyse liegt u.a. in der einfachen parallelen Interpretation der Lagebeziehungen von Arten und Aufnahmen zueinander. Weitere Erläuterungen und Beispiele zu der Korrespondenzanalyse finden sich z. B. bei GlaVAC (1996: 167ff.) oder bei Richter (1997: 98ff.).

\section{Ergebnisse und Interpretation}

In Abb. 4 ist das Ergebnis der Korrespondenzanalyse für den Datensatz in Form eines beschrifteten Ordinationsdiagrammes zu sehen: Dargestellt ist das Diagramm der ersten beiden Achsen mit der Lage aller Aufnahmen und einiger exemplarischer Arten, die als Kreuze dargestellt sind. Die unterschiedlichen Symbole für die Aufnahmeflächen geben das «Alter» einer Fläche an, d.h. die Zahl der Jahre seit dem Eisfreiwerden zum Zeitpunkt der pflanzensoziologischen Aufnahme.

Die Aufnahmen reihen sich entsprechend ihrer flori- stischen Ähnlichkeit bzw. Unähnlichkeit entlang eines durchgehenden Gradienten auf, welchem auch die dargestellten Arten folgen. Die Form entspricht einer Parabel; dies weist auf einen sog. "Arch» hin, einen mathematischen Effekt, der auftritt, wenn der Datensatz einen dominierenden Gradienten enthält, welcher den grössten Teil der floristischen Varianz «verursacht». Hierbei stellt die zweite Achse eine nahezu quadratischen Funktion der ersten Achse dar. Dies bedeutet auch, dass eine Interpretation der zweiten Achse nicht sinnvoll ist. Mit der Darstellung der ersten gegen die zweite Achse ist eine erschöpfende Darstellung erreicht, das Einbeziehen weiterer Achsen in die Darstellung würde keine zusätzlichen Informationen liefern.

Betrachtet man den Verlauf des Gradienten, so fällt auf, dass er im II. Quadranten links oben mit 0 bis 10 Jahre alten Aufnahmeflächen beginnt, begleitet von Pionierarten wie Linaria alpina oder Sagina saginoides. Weitere frühe Pionierarten wie Saxifraga aizoides oder Epilobium fleischeri besitzen einen Schwerpunkt ihres Auftretens erst im späteren Verlauf des 


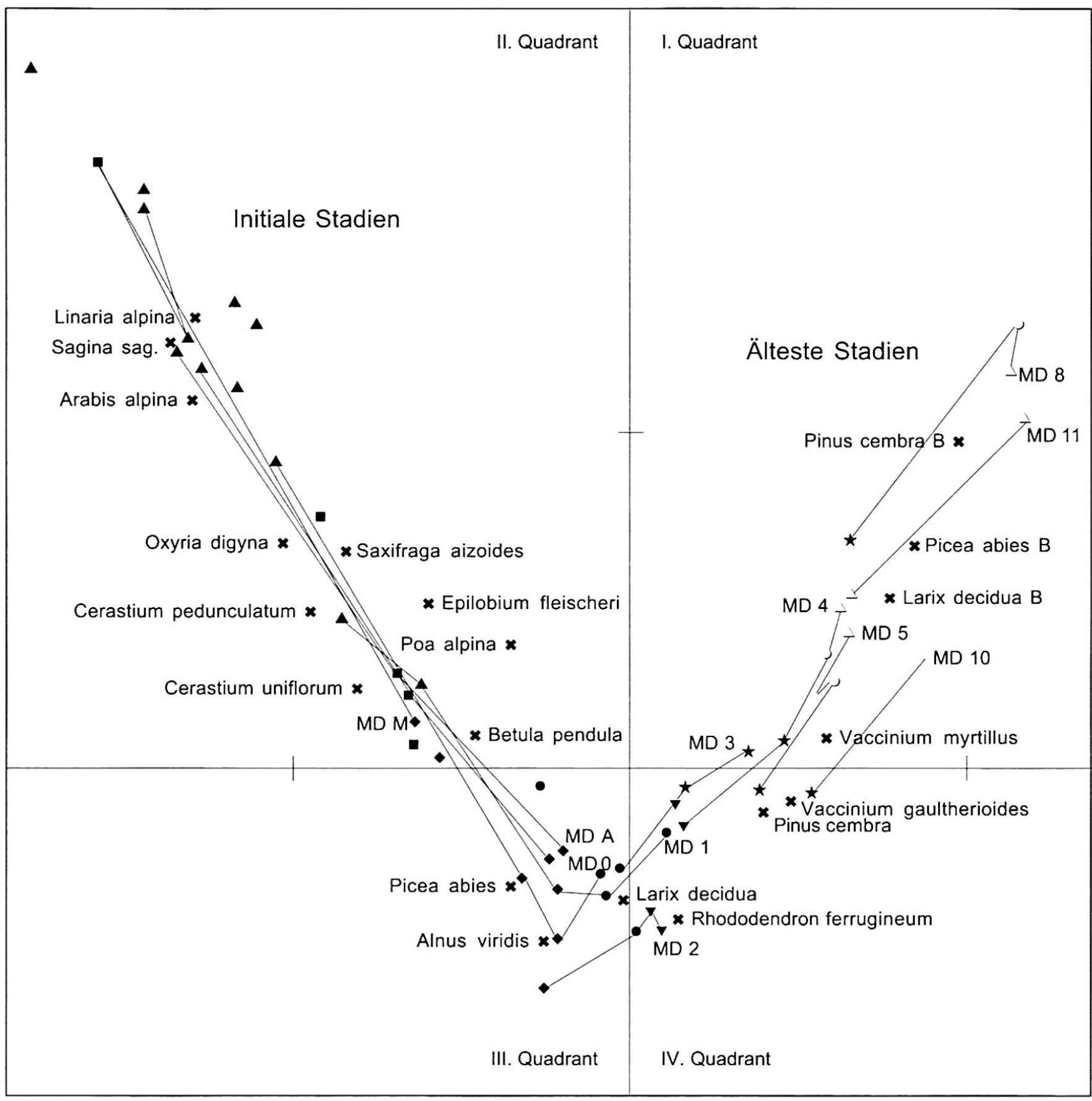

* Die Kreuze entsprechen dem Schwerpunkt des Auftretens genannter Arten. Die übrigen Symbole entsprechen den Aufnahmeflächen. Ihre Form gibt die Zahl der Jahre seit Eisfreiwerden zum Zeitpunkt der Aufnahme an:
$\Delta$ - $0-10$
$11-20$
$21-40$
$41-60$
$61-80$
$\star 81-100$
J $101-120$
$\rightarrow 121-140$
- Jeweils einer Dauerfläche angehörende Aufnahmen sind durch schwarze Striche miteinander verbunden.

Abb. 4: Ordinationsdiagramm einer Korrespondenzanalyse anhand von 51 Aufnahmen mit 170 Arten von 1944 bis 1998

Scatterplot of the correspondence analysis of 51 sites with 170 species from 1944 to 1998

"Scatterplot" de l'analyse des correspondances de 51 sites avec 170 espèces, inventoriés de 1944 à 1998

Graphik: S. ReHBERGER 
Gradienten. Dies darf nicht dahingehend interpretiert werden, dass diese Arten erst nach Pionieren wie zum Beispiel Linaria alpina im Sukzessionsverlauf auftauchen würden. Es kennzeichnet vielmehr eine Art «Beharrungsvermögen»: Sie sind auch in späteren Entwicklungsstadien noch vertreten, weil sie der Konkurrenz neu auftretender Arten standhalten oder ihr ausweichen können. Welche Strategien dafür verantwortlich sind, hat STöcKLIN (1999) u.a. am Beispiel von Epilobium fleischeri gezeigt.

Im III. Quadranten befinden sich mehrheitlich 21 bis 60 Jahre alte Aufnahmeflächen und dazu der Schwerpunkt des Auftretens von jüngeren Bäumen wie Larix decidua oder Picea abies in der Krautschicht. Im IV. Quadranten dominieren 61 bis 100 Jahre alte Flächen, begleitet von Zwergstraucharten wie Rhododendron ferrugineum oder Vaccinium gaultherioides sowie der verzögert hinzutretenden Arve Pinus cembra. Im I. Quadranten liegen vor allem die Flächen, die zum Zeitpunkt der Aufnahme bereits mehr als 100 Jahre eisfrei waren. Hier haben Larix decidua, Picea abies und Pinus cembra in der Baumschicht den Schwerpunkt ihres Auftretens. Zwar sind damit in der Baumschicht bereits die beiden namengebenden Baumarten des Larici-Pinetum cembrae vorhanden, jedoch fehlen in der Krautschicht noch wichtige Vertreter wie z. B. das Wollige Reitgras Calamagrostis villosa, und auch die Deckungswerte der Arten in der Strauchschicht weichen bisher noch erheblich von denen des direkt benachbarten Lärchen-Arven-Waldes auf mehrere tausend Jahre eisfreiem Substrat ab. Die Geschwindigkeit der Sukzession hat sich zwar merklich verringert und die Flächen sind bereits artenärmer als jene mittleren Alters, jedoch wird es vermutlich noch lange dauern, bis auf den ältesten Moränenflächen eine ähnliche Waldstruktur wie in den Flächen des Lärchen-Arven-Waldes zu beobachten ist.

Es ist in der Konsequenz der dargestellten Ergebnisse naheliegend, den dominierenden Gradienten als Zeitachse zu interpretieren. Gewissheit darüber vermittelt das Einbeziehen der Dauerflächen-Informationen in die Betrachtung: In Abb. 4 sind alle Aufnahmen, die von einer identischen Fläche zu unterschiedlichen Zeitpunkten stammen, durch Linien miteinander verbunden. Zudem sind die Aufnahmen von 1998, welche den aktuellen Stand der Entwicklung markieren, mit ihrer jeweiligen Flächenbezeichnung entsprechend Tab. 1 versehen.

Die dabei beobachteten klaren Entwicklungslinien auf konkreten Flächen decken sich weitgehend mit dem aus der Korrespondenzanalyse erhaltenen Zeitgradienten. Dies bestätigt LüDI (1945), der mangels anderer Daten die Aufnahmen aus den unterschiedlichen Flächen im Sinne der Chronosequenz ausgewertet hat; seine aus dieser Interpretation gewonnene Vorstellung der Vegetationsentwicklung ist weitgehend korrekt. Allerdings werden auch die Grenzen seines Modells sichtbar: Einzelne Flächen reagieren in verschiedenen Phasen ihrer Entwicklung individuell auf spezifische Faktoren.

So macht die Fläche MD 8 von 1971 bis 1998 eine eigene Entwicklung durch, die tendenziell rückwärts entlang des Gradienten deutet. Ähnliches passierte der Fläche MD 5 zwischen 1971 und 1981 und der Fläche MD M relativ früh in ihrer Entwicklung. Auffällig ist auch der Knick in der Entwicklung von MD 2, die sich zwischen 1981 und 1998 «quer» zu dem Hauptgradienten entwickelt hat. Vergleicht man das Arteninventar der Fläche MD 2 in den verschiedenen Jahren, dann ergibt sich, dass seit 1971 mehrere strauchige Weidenarten ausgefallen sind, so zum Beispiel Salix hastata, Salix purpurea oder Salix fodetida, andere wie Salix helvetica zeigen deutliche Verbiss-Spuren.

Die Fläche MD 2 wird aufgrund ihrer Nähe zu einem feuchten Bestand von Alnus viridis von Gems- und Rothirschwechseln durchquert. $\mathrm{Da}$ in den vergangenen 20 Jahren die Zahl der Hirsche mangels Bejagung oder natürlicher Feinde stark gestiegen ist, sind überall deutliche Verbiss-Schäden an den Bäumen und Sträuchern zu beobachten. Es ist zu vermuten, dass in diesem Fall Hirsche den Sukzessionsverlauf beeinflussen.

Die Fläche MD M ist leicht geneigt, und auf ihr fanden offenbar mehrere Rutschungen statt, die nicht nur zum bereits genannten Verlust zweier Pflöcke führten, sondern auch zu Verlust oder Verringerung in der Dekkung einiger Arten, so dass auch von einem Rückschlag bzw. einer Retrogression gesprochen werden kann.

Da ein grosser Teil der Moränenflächen innerhalb des Untersuchungsgebietes deutliche Neigungen aufweist, ist eine Verallgemeinerung der Erkenntnisse aus den ebenen Dauerflächen nur beschränkt möglich. Natürliche Störungsereignisse wie Rutschungen, Steinschlag, Schneedruck und Oberflächenerosion etc. gehören zu den typischen Entwicklungsfaktoren der Vegetation auf Moränenflächen. Sie müssen bei der Betrachtung der Vegetationsentwicklung auf Hangflächen des Untersuchungsgebietes unbedingt miteinbezogen werden.

\section{Fazit}

Die bis in die heutige Zeit immer wieder gestellte Frage, ob die Arbeit mit Dauerflächen oder die mit Chronosequenzen ein treffenderes Bild der Primärsukzession zu liefern vermag, lässt sich pauschal nicht beantworten. Beide Ansätze haben für sich genommen eine Reihe methodischer Nachteile und entfalten optimale 
Interpretationsmöglichkeiten erst in der Kombination.

Dauerflächen sind zum Beispiel problematisch, wenn die ständige Umschichtung des Substrates eine Markierung der Fläche unmöglich macht. Eine Alternative ist in so einem Fall das Einmessen der Begrenzungen, um z.B. auch steilere Hangflächen in die Untersuchungen miteinzubeziehen. Da Dauerflächen zugleich zeitaufwendig in der Bearbeitung sind, ist es zuweilen schwierig, ausreichend viele Flächen zu wählen, in denen alle relevanten Faktoren der Primärsukzession wirken. Dies gilt zum Beispiel, wenn Zufallsereignisse zu vorübergehend divergierenden Entwicklungen führen.

Der Arbeit mit Chronosequenzen steht der Einwand gegenüber, dass die vorausgesetzte Konstanz der Umweltbedingungen nicht gegeben ist, angefangen bei Stickstoffdepositionen bis hin zu den üblichen leichten Veränderungen des Klimas. Auch sind die aus ihr gewonnenen Gradienten zum Teil hypothetischen und summierenden Charakters. Man erkennt zwar das grobe Muster eines Entwicklungsverlaufes und findet auch Hinweise auf Rückschläge oder Sonderentwicklungen. Aber ob und wie diese konkret auf einer Fläche stattgefunden haben, können Chronosequenzen nicht beantworten.

Es spricht also viel dafür, das eine zu tun, ohne das andere zu lassen, und eine Kombination beider Ansätze zu wählen, wo immer dies möglich und durch die Fragestellung nahegelegt ist. Die Arbeit mit Dauerflächen verlangt ohnehin viel Geduld oder wie im vorliegenden Fall vorausschauendes Denken vorheriger Generationen, von dessen spannenden Ergebnissen wir heute profitieren.

\section{Ausblick}

Forschungsbedarf besteht für das Aletschgebiet im Bereich der Hangflächen, die bisher aus allen Untersuchungen ausgeklammert wurden. Erste Schritte in diese Richtung wurden im Sommer 1999 unternommen. Ziel ist, zur Klärung der Frage beizutragen, inwieweit die durch Störungen bzw. fortgesetzte dynamische Vorgänge beeinflussten Hangflächen eine lediglich verzögerte Entwicklung im Sinne kleiner Retrogressionen durchlaufen oder auch Sonderentwicklungen abweichend von dem skizzierten Gradienten beobachtbar sind.

\section{Dank}

Die vorliegende Arbeit wurde freundlicherweise unterstützt durch: Pro Natura-Zentrum Aletsch, Prof. Jean
Louis Richard, Neuenburg, und Prof. Arno Bogenrieder, Freiburg im Breisgau.

\section{Literatur}

Barkman, J.J., Doing, H. \& S. Segal (1964): Kritische Bemerkungen und Vorschläge zur quantitativen Vegetationsanalyse. - In: Acta Botanica Neerlandica 13: 394-419.

Bertram, J. (2000): Moosvegetation und Moosflora des Reservates Aletschwald. - = Les Cahiers des sciences naturelles 4, Sion: 1-143.

BÖHMER, H.J. (1999): Vegetationsdynamik im Hochgebirge unter dem Einfluss natürlicher Störungen. - = Dissertationes Botanicae 311, Stuttgart: 1-180.

Braun-Blanquet (1928): Pflanzensoziologie. Grundzüge der Vegetationskunde. - Berlin: Springer.

Burga, C.A. (1999): Vegetation developement on the glacier forefield Morteratsch (Switzerland). - In: Applied Vegetation Science 2:17-24.

COAZ, J. (1887): Erste Ansiedlung phanerogamischer Pflanzen auf von Gletschern verlassenem Boden. In: Mitteilungen der Naturforschenden Gesellschaft Bern: 3-12.

COOPER, W.S. (1923): The recent ecological history of Glacier Bay, Alaska. - In: Ecology 23: 93-128, 223-246.

EgGenberg, S. (1996): Verjüngung an der Waldgrenze des Aletschreservates (Wallis, Schweiz). - In: VITTOZ, P., Theurillat, J.-P., Zimmermann, K. \& J.D. GallanDAT (eds) (1996): Volume jubilaire J.-L. Richard. - = Dissertationes Botanicae 258, Stuttgart: 115-124.

EGGER, G. (1997): Standortsdynamik und Sukzession der Flechtenbesiedlung im Winklkees-Vorfeld des Seebachtales (Nationalpark Hohe Tauern, Österreich). - In: Wissenschaftliche Mitteilungen aus dem Nationalpark Hohe Tauern 3: 37-65.

ERSCHBAmER, B. (1997): Climate warming and plant growth on glacial retreats. - In: Supplementum Revue Valdôtaine d'Histoire Naturelle 51:219-224.

Erschbamer, B., Niederfriniger-Schlag, R. \& E. KNERINGer (1997): Seed dispersal, seed bank and establishment of seedlings on glacial retreats. - In: Supplementum Revue Valdôtaine d'Histoire Naturelle 51:225-232.

Erschbamer, B., Bitterlich, W. \& C. RafFl (1999): Die Vegetation als Indikator für die Bodenbildung im Gletschervorfeld des Rotmossferners (Obergurgl, Ötztal, Nordtirol). - In: Berichte des NaturwissenschaftlichMedizinischen Vereins Innsbruck 86: 107-122.

GlaVAC, V. (1996): Vegetationsökologie. Grundfragen, Aufgaben, Methoden. - Jena: Fischer.

Holzhauser, H. (1984): Zur Geschichte des Aletschund des Fieschergletschers. $-=$ Physische Geographie Vol. 13, Zürich: 1-448.

Holzhauser, H. (1988): Methoden zur Rekonstruktion von Gletscherschwankungen. Der Grosse Aletsch- 
gletscher. - In: Zumbühl, H.J. \& H. Holzhauser: Alpengletscher in der Kleinen Eiszeit. - Die Alpen 64 (3): 135-165.

HolzHaUSER, H. (1995): Gletscherschwankungen innerhalb der letzten 3200 Jahre am Beispiel des Grossen Aletsch- und des Gornergletschers. - In: Schweizerische Gletscherkommission (Hrsg.): Gletscher im ständigen Wandel. Bericht über das Jubiläums-Symposium der Schweizerischen Gletscherkommission 1993 Verbier (VS). - = In: Publikationen der Schweizerischen Akademie der Naturwissenschaften 6, Zürich: 101-122.

Krause, H.G. \& B. Frenzel (1997): Die Entwicklung der Vegetation eines zentralalpinen Gletschervorfeldes seit dem Jahr 1957. - In: Jahrbuch des Vereins zum Schutz der Bergwelt 62: 189-226.

LüDI, W. (1945): Besiedlung und Vegetationsentwicklung auf den jungen Seitenmoränen des Grossen Aletschgletschers, mit einem Vergleich der Besiedlung im Vorfeld des Rhonegletschers und des Oberen Grindelwaldgletschers. - In: Berichte des Geobotanischen Forschungsinstitutes Rübel Zürich 1944: 35-112.

LüDI, W. (1950): Die Pflanzenwelt des Aletschreservates bei Brig (Wallis). - In: Bulletin Murithienne 67: 122-179.

MatThews, J.A. (1992): The ecology of recently deglaciated terrain: a geoecological approach to glacier forelands and primary succession. $-=$ Cambridge Studies in Ecology, Cambridge: 1-386.

Morier-Genoud, P. (1982): Contribution à l'étude de la dynamique de la végétation sur les jeunes moraines du grand glacier d'Aletsch. - Unveröffentlichte Lizentiatsarbeit, Université Neuchâtel: 1-59.

KretZ, J. (1972): Kontrolle und Aufnahme der Vegetation in den Dauerflächen auf dem Jungmoränengebiet des Aletschreservates. - Unveröffentlichte Abschlussarbeit für das luzernerische Sekundarlehrerdiplom, Luzern: 1-62.

PicketT, S.T.A. (1989): Space-for-time substitution as an alternative to long-term studies. - In: LiKENS, E.D. (Hrsg.): Long-Term-Studies in Ecology. - Berlin: Springer: 110-135.

RichARD, J.-L. (1968): Les groupements végétaux de la Réserve d'Aletsch (Valais, Suisse). - = Beiträge zur Geobotanischen Landesaufnahme der Schweiz 51, Bern: 1-30.

RiCHARD, J.-L. (1973): Dynamique de la végétation au bord du grand glacier d'Aletsch (Alpes Suisses). In: Berichte der Schweizerischen Botanischen Gesellschaft 83: 159-174.

RiCHARD, J.-L. (1987): Dynamique de la végétation sur les marges glaciaires récentes de la réserve d'Aletsch (Alpes valaisannes). - In: Botanica Helvetica 97: 265-275.

Richter, M. (1994): Die Pflanzensukzession im Vorfeld des Tschierva-Gletschers/Oberengadin. - In: Geoökodynamik 15: 55-88.
Richter, M. (1997): Allgemeine Pflanzengeographie. Stuttgart: Teubner.

SChubiger-Bossard, C.M. (1988): Die Vegetation des Rhonegletschers, ihre Sukzession und naturräumliche Gliederung. - = Beiträge zur Geobotanischen Landesaufnahme der Schweiz 64, Teufen: 1-228.

STÖCKLIN, J. \& E. BÄUMLER (1996): Seed rain, seedling establishment and clonal growth strategies on a glacier foreland. - In: Journal of Vegetation Science 7:45-56.

STöCKLIN, J. (1999): Differences in life history traits of related Epilobium species: clonality, seed size and seed number. - In: Folia Geobotanica 34: 7-18.

Theurillat, J.P. (1992): Etude et cartographie du paysage végétal (symphytocoenologie) dans la région d'Aletsch (Valais, Suisse). - = Beiträge zur Geobotanischen Landesaufnahme der Schweiz 68, Teufen: 1-384. Thioulouse, J., Chessel, D., Dolédec, S. \& J.M. OLIVIER (1997): ADE-4: a multivariate analysis and graphical display software. - In: Statistics and Computing 7: 75-83.

Thioulouse, J., Chessel, D. \& S. Dolédec (1998): Manual for ADE-4 for Apple Macintosh. - Zusammen mit dem Programm frei verfügbar in der Programmbibliothek der Universität Lyon. http://pbil.univ-lyon1.fr/ ADE-4/ (29.02.2000)

\section{Zusammenfassung: Vegetationsdynamik auf Moränendauerflächen des Grossen Aletschgletschers im Wallis - Erste Ergebnisse aus den Neuaufnahmen der 54jährigen Flächen von Lüdi}

Die von LüDI (1945) am Rande des Grossen Aletschgletschers angelegten Dauerflächen auf neuzeitlichen Moränen wurden 1998 pflanzensoziologisch neu aufgenommen und mit den bereits vorliegenden Daten aus den Jahren 1944, 1971 und 1981 im Rahmen einer Korrespondenzanalyse zusammenfassend ausgewertet. Das Ergebnis bestätigt weitgehend die Vorstellungen, welche bereits durch die Methode der «space-fortime-substitution» gewonnen wurden. Zugleich weisen die Ergebnisse auf die Grenzen dieser Methode hin, da die konkrete Entwicklung auf einzelnen Flächen individuelle Züge trägt, welche erst durch die Methode der Dauerflächen im Ordinationsdiagramm sichtbar gemacht werden können.

\section{Summary: Vegetation Dynamics on Moraine Fields of the Great Aletsch Glacier (Valais, Switzerland) - First results of renewed investigation of permanent plots established in 1944 by Lüdi}

Renewed investigation is currently under way on permanent plots established by LüDI in 1945 on moraine fields bordering the Great Aletsch Glacier. The plots were set up according to the BRAUN-BLANQUET method. The phytosociological data from 1944, 1971, 1981 and 1998 were processed using correspondence analysis. The results indicate that the model of primary suc- 
cession derived from a «space-for-time-substitution» seems to be correct. However, the individual direction of development on some of the plots, made apparent by means of scatterplots, points to limitations in the above-mentioned approach.

\section{Résumé: La dynamique végétale sur les surfaces} morainiques permanentes du «Grosser Aletschgletscher» (Valais) - Premiers résultats des nouveaux relevés effectués sur les surfaces de Lüdi qui remontent à 54 années

Les surfaces permanentes établies par LüDI (1945) au bord du «Grosser Aletschgletscher» sur des moraines contemporaines ont été donné bien à de nouveaux relevés phytosociologiques en 1998 et comparées de façon synthétique avec les données déjà disponibles pour les années 1944, 1971 et 1981, dans le cadre d'une analyse des correspondances. Les résultats obtenus confirment amplement les représentations déjà élaborées selon la méthode «space-for-time-substitution». Ils permettent en même temps de prendre con- science des limites de cette méthode, dans la mesure où l'évolution concrète de certaines surfaces est marquée par des traits individuels, qui ne sont perceptibles que par le recours au «scatterplot» de la méthode des surfaces permanentes.

Diplom-Biologe Silvan Rehberger,Seb.-Kneipp-Strasse 7, D-79104 Freiburg im Breisgau.

e-mail:sr@youngbrain.com

\section{Manuskripteingang/received/manuscrit entré le} 3.5.2002

Annahme zum Druck/accepted for publication/accepté pour l'impression: 20.9.2002 